\title{
Anthropometry, DXA, and leptin reflect subcutaneous but not visceral abdominal adipose tissue on MRI in 197 healthy adolescents
}

\author{
Jeanette Tinggaard ${ }^{1}$, Casper P. Hagen ${ }^{1}$, Anders N. Christensen ${ }^{2}$, Annette Mouritsen ${ }^{1}$, Mikkel G. Mieritz ${ }^{1}$, \\ Christine Wohlfahrt-Veje ${ }^{1}$, Jørn W. Helge ${ }^{3}$, Thomas N. Beck ${ }^{3}$, Eva Fallentin ${ }^{4}$, Rasmus Larsen ${ }^{2}$, Rikke B. Jensen ${ }^{1}$, Anders Juul ${ }^{1}$ \\ and Katharina M. Main ${ }^{1}$
}

BACKGROUND: Abdominal fat distribution is associated with the development of cardio-metabolic disease independently of body mass index (BMI). We assessed anthropometry, serum adipokines, and DXA as markers of abdominal subcutaneous adipose tissue (SAT) and visceral adipose tissue (VAT) using magnetic resonance imaging (MRI).

METHODS: We performed a cross-sectional study that included 197 healthy adolescents (114 boys) aged 10-15 years nested within a longitudinal population-based cohort. Clinical examination, blood sampling, DXA, and abdominal MRI were performed. SAT\% and VAT\% were adjusted to total abdominal volume.

RESULTS: Girls had a higher SAT\% than did boys in early and late puberty (16 vs. $13 \%, P<0.01$ and 20 vs. $15 \%$, $P=0.001$, respectively), whereas VAT\% was comparable (7\% in both genders, independently of puberty). DXA android fat\% (standard deviation score (SDS)), suprailiac skinfold thickness (SDS), leptin, BMI (SDS), waist-to-height ratio $(\mathrm{WHtR})$, and waist circumference (SDS) correlated strongly with SAT\% (descending order: $r=0.90-0.55$, all $P<0.001$ ) but weakly with VAT\% $(r=0.49-0.06)$. Suprailiac skinfold was the best anthropometric marker of SAT\% (girls: $R^{2}=48.6 \%$, boys: $\left.R^{2}=65 \%, P<0.001\right)$ and VAT\% in boys $\left(R^{2}=16.4 \%, P<0.001\right)$. WHtR was the best marker of VAT\% in girls $\left(R^{2}=7.6 \%\right.$, $P=0.007)$.

CONCLUSIONS: Healthy girls have a higher SAT\% than do boys, whereas VAT\% is comparable, independently of puberty. Anthropometry and circulating leptin are valid markers of SAT $\%$, but not of VAT\%.

C hildhood obesity is associated with an increased risk of developing type 2 diabetes and cardiovascular disease (CVD) in adulthood (1). Overweight and obesity during childhood is mostly defined by age- and sex-standardized body mass index (BMI) cutoffs (2). However, normal-weight children, as defined by BMI, may also develop metabolic disturbances. These children are characterized by an unhealthy body fat distribution (3).

Abdominal adiposity is strongly related to the development of cardiovascular and metabolic diseases in adulthood regardless of BMI (4). In particular, increased visceral adipose tissue (VAT) compared with subcutaneous adipose tissue (SAT) in the abdominal region is unhealthy. As BMI is a crude marker of body fat distribution, in particular VAT, (5) other markers of body fat are needed to identify children at risk for abdominal adiposity who may benefit from early intervention. This may help to reduce the worldwide increasing prevalence of type 2 diabetes and CVD. The "metabolic unhealthy normal weight" phenotype is also evident during childhood, although knowledge on SAT and VAT in these children is sparse. Whether SAT and VAT can be assessed by simple methods in normal-weight children as compared with obese children is not well established.

From adult studies, it is well known that men deposit more abdominal adipose tissue than do women, especially VAT. Concordantly, cardio-metabolic disease is more frequent among men than among women. Visceral and subcutaneous adipocytes derive from separate progenitor cells, express different genes, and exert separate endocrine functions (6). An increased amount of VAT among boys as compared with girls potentially relates to cardio-metabolic risk later in life. Thus, early intervention may be targeted more specifically toward boys than toward girls. It is not clear whether a gender difference in the amount of VAT or a gender difference in the VAT-to-SAT ratio is apparent already during childhood.

A highly useful method for measuring abdominal VAT and SAT is magnetic resonance imaging (MRI), because it provides high-resolution images without radiation, as compared to computerized tomography (7). However, an MRI is time-consuming and expensive and demands good compliance, which limits the use of MRI in large cohort studies and in clinical practice. Hence, population-based

\footnotetext{
${ }^{1}$ Department of Growth and Reproduction, Rigshospitalet, Copenhagen University Hospital, Copenhagen, Denmark; ${ }^{2}$ DTU Compute, Technical University of Denmark, Kgs. Lyngby, Denmark; ${ }^{3}$ Department of Biomedical Sciences, Faculty of Health and Medical Sciences, University of Copenhagen, Copenhagen, Denmark; ${ }^{4}$ Department of Radiology, Rigshospitalet, Copenhagen University Hospital, Copenhagen, Denmark. Correspondence: Jeanette Tinggaard, (jeanette.tinggaard.01@regionh.dk) 
studies on abdominal fat using MRI in children are limited. Whole-body dual-X-ray absorptiometry (DXA) measures total and regional fat percentage, including the android region, which corresponds to the abdominal region. However, a distinction between abdominal adipose tissue compartments is not possible in children, as it is in adults. Whether the DXA android region can be used as a marker of VAT in children is largely unknown.

Waist circumference (WC), waist-to-height ratio (WHtR), and suprailiac skinfold measurement may be better markers of abdominal adiposity than BMI (8). WC-for-age is widely used as a marker of abdominal adiposity and metabolic health in children (9). Although most studies agree that WC reflects SAT rather than VAT in children $(10,11)$, this needs further investigation because of study differences in, e.g., child age and imaging method. In children, the WHtR has been found to predict cardio-metabolic health, but the correlation to VAT is largely unknown (12). Similarly, limited data on the usefulness of suprailiac skinfold thickness as a marker of VAT exist. Simple anthropometry is inexpensive, rapid, and feasible in primary care units as well as in tertiary care.

In addition to anthropometry, biomarkers of adipose tissue may be useful in assessing fat distribution. Circulating adiponectin has an insulin-sensitizing effect and is inversely associated with visceral adipose tissue in obese children (13), whereas leptin regulates the energy balance and is positively associated with BMI and adiposity (14). However, knowledge on the usefulness of these circulating adipokines as markers of SAT and VAT in normal-weight children is sparse.

In this study, we have measured SAT and VAT using MRI in normal-weight children. The purpose of the current study was to identify a marker for SAT and VAT among normalweight children. We hypothesized that SAT and VAT determined by MRI varied within normal-weight children and that gender differences in abdominal fat were already evident in childhood.

\section{METHODS}

\section{Study Population}

This study included 197 Caucasian children ( $n=114$ boys and $n=83$ girls) who were a part of a population-based longitudinal birth cohort study that included 2,788 mothers and their children who were born between 1997 and 2001 (15). Of them, 1,293 children agreed to participate in the pubertal follow-up from August 2009, and 256 young adolescents ( $n=131$ boys and $n=125$ girls) (age 1014.8 years) agreed to undergo an MRI of the abdomen in addition to the standard protocol. The participants who were invited for the MRI were selected on the basis of a high frequency of prior clinical examinations (a minimum of five visits) during childhood.

\section{Methods}

At a single visit to the department during the pubertal follow-up we performed a clinical examination, venous blood sampling, wholebody DXA, and an MRI of the abdomen. The abdominal MRI protocol was performed in 228 children ( $n=119$ boys and $n=109$ girls) (Supplementary Figure S1 online). However, 31 MRI scans were later excluded because of artifacts $(n=3)$, incorrect scan area $(n=4)$, or different technical MRI protocol $(n=24$, all girls). Anthropometric measurements were available in all remaining 197 participants, pubertal stage was available in 186/197 (94\%), and DXA in 196/197 (99\%). Gestational age (GA) was determined by obstetric ultrasound during early pregnancy. Information on birth weight and gestational age at birth was obtained from birth records. Genderspecific birth weight adjusted for GA was calculated according to Marsal and referred to as birth weight standard deviation score (BW SDS) (16).

\section{Clinical Examination}

The clinical examination included measurement of height to the nearest mm using a Harpenden Stadiometer (Holtain, Crymych, UK) and weight to the nearest $0.1 \mathrm{~kg}$ using electronic scales (SECA delta model 707, Seca, Hamburg, Germany, and Bisco Model PERS 200, Bisco, Farum, Denmark). Waist circumference (WC) to the nearest $\mathrm{mm}$ was measured using a flexible measurement tape parallel to the floor at the midpoint between the ribs and the top of the iliac crest. Skinfold thickness (triceps, biceps, subscapular, and suprailiac) was measured to the nearest mm using a Harpenden calliper (John Bull, British Indicators, Weybridge, UK). All anthropometric measurements were taken in triplet and the mean was calculated. Intra- and inter-observer coefficients of variability for skinfold measurement have previously been published (15). BMI was calculated as weight $(\mathrm{kg}) /$ height $^{2}\left(\mathrm{~m}^{2}\right)$, and WHtR was calculated as WC $(\mathrm{cm})$ divided by height $(\mathrm{cm})$. Pubertal assessment was performed by inspection according to Tanner with additional breast palpation in girls to distinguish glandular from adipose tissue

\section{Dual X-Ray Absorptiometry}

A whole-body DXA scan was performed (Lunar Prodigy, GE Healthcare, Madison, WI, using enCORE software, version 14.10.022) with subjects wearing standardized light clothing. The regions and tissue of interest in this study were total and android fat mass and percentage. The android region corresponded to the abdominal area, with the pelvis line as the lower demarcation and the upper demarcation taken at $20 \%$ of the distance between the iliac crest and the neck. A single trained investigator checked all scans for positioning.

\section{MRI of the Abdomen}

MRI was performed with a 3-Tesla MRI (Magnetom Verio; Siemens AG, Erlangen, Germany). We used a 32-channel receiver array coil. MRI of the abdomen was part of a comprehensive MRI study including adrenals, (17) breasts (18), and ovaries (19). For fat quantification, a 3D T1-weighted VIBE Dixon sequence, providing water and fat images, was performed during breath-hold for $19 \mathrm{~s}$ in the transverse plane from the upper level of lumbar vertebrae 1 to the lower level of lumbar vertebrae 4 . The scan parameters were as follows: TR/TE1/TE2 5.49/2.45/3.675 ms, flip angle $9^{\circ}$, FOV $350 \mathrm{~mm}$, in plane matrix $320 \times 214$, and a slice thickness of $4 \mathrm{~mm}$, providing a voxel size of $1.6 \times 1.1 \times 4 \mathrm{~mm}$. Automated segmentation was used to assess the amount of abdominal fat. The varying intensity (the bias field) of abdominal fat tissue in the raw DIXON fat image (Figure 1a) was corrected for by applying sampling points to the image, followed by fitting a sheet called thin plate spline (TPS). After logtransformation and subtraction, this resulted in a homogenous intensity (Figure 1b) (20). The segmentation was done using graph cuts after a radial unrolling of the images to find the outer edge skin/ air and inner edge musculature/abdomen (21), followed by an Otsu threshold that automatically finds the cutoff of the gray-level histograms of the MRI picture processing that distinguishes between fat and non-fat (22) (Figure 1c). In this way, the total abdominal volume $(\mathrm{ml})$, VAT volume $(\mathrm{ml})$, and SAT volume $(\mathrm{ml})$ were determined (Figure 1d). Automated segmentation was verified visually, and potential artifacts or aberrations in the scan area were described by an expert in MRI data analysis (AC). Furthermore, potential bright signaling from bone marrow was corrected for manually.

Total adipose tissue (TAT) volume $(\mathrm{ml})$ of the abdomen was calculated as the sum of VAT and SAT. TAT, SAT, and VAT percentages (TAT\%, SAT\%, and VAT\%) were calculated as TAT, 

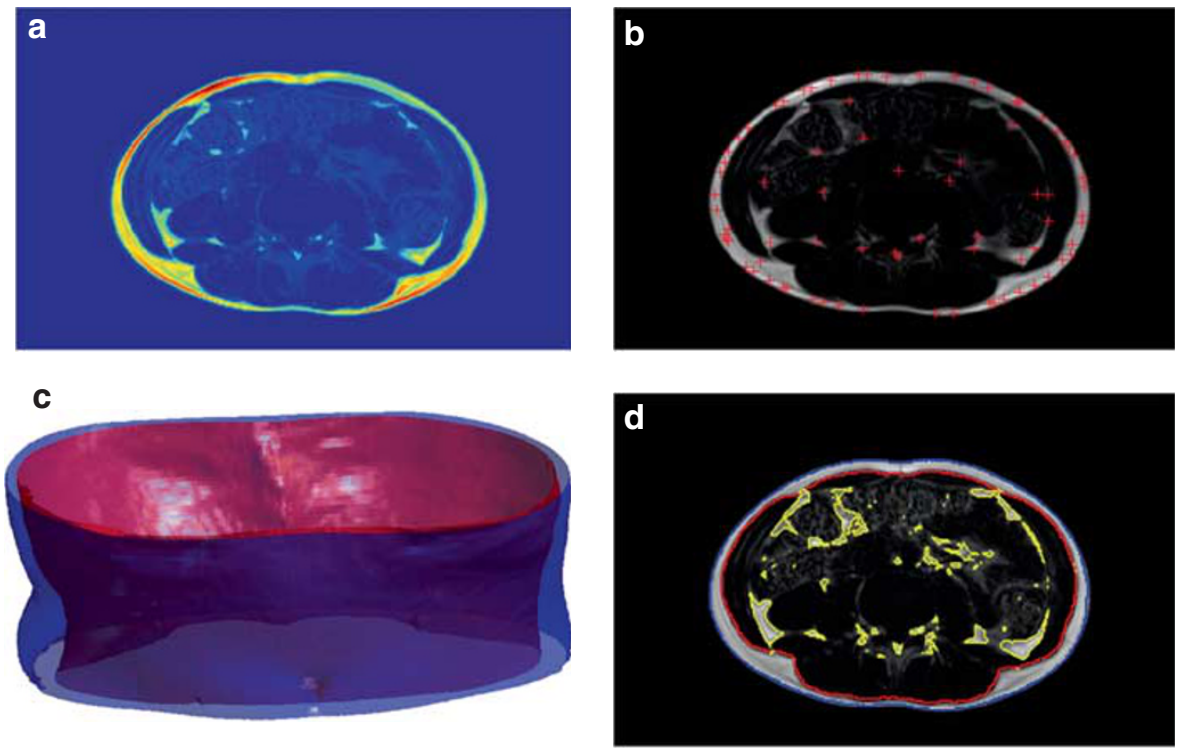

Figure 1. Abdominal fat imaging by MRI. (a) Raw DIXON fat image. The intensity (the bias field) of abdominal fat tissue varies from light blue to yellow, green, and red. (b) Raw DIXON fat image. Sampling points in fat tissue (red crosses) followed by a thin plate spline were used to correct for the varying intensity of abdominal fat and obtain a homogenous intensity of fat (gray color). (c) The MRl-abdominal region. Cut graphs were used to find the outer edge skin/air (blue demarcation)) and inner edge musculature/abdomen (red demarcation). (d) Final segmentation: total abdominal volume (volume within the blue demarcation), intraabdominal fat volume (volume within the yellow demarcations), and subcutaneous abdominal volume (volume within the blue and red demarcations).

SAT, or VAT volume divided by total abdominal volume, respectively. These percentages adjust for differences in scan area and volume due to variation in height. Furthermore, a VAT-to-SAT (VAT/SAT) ratio was calculated.

\section{Laboratory Analyses}

A non-fasting blood sample was drawn from an antecubital vein between 0800 hours and 1500 hours. They were clotted, centrifuged, and immediately stored at $20^{\circ} \mathrm{C}$ until analyses were performed. Total adiponectin was measured by means of a radio-immunoassay (RIA) (EMD Millipore Corporation, MA) with detection limits of $1 \mathrm{ng} / \mathrm{ml}$. Intra- and inter-assay coefficients of variations (CVs) were 5.91 and $5.73 \%$, respectively.

Leptin was measured by means of ELISA (Qiantikine ELISA, R\&D Systems, Minneapolis, MN) with detection limits of $7.8 \mathrm{pg} / \mathrm{ml}$. Intraand inter-assay CVs were 8.31 and $6.86 \%$, respectively. Leptin-toadiponectin ratio (LAR) was calculated as leptin $(\mathrm{pg} / \mathrm{ml})$ divided by adiponectin $(\mathrm{ng} / \mathrm{ml})$.

\section{Statistics}

Age- and sex-specific standard deviation scores (SDS) were calculated according to national references $(15,23)$. Android fat\% SDS was calculated from concomitantly developed reference data based on 982 DXA scans ( $n=556$ boys) of children/adolescents participating in the pubertal follow-up (age 8-15 years), see Supplementary Figure S2, and Supplementary Table S1. The generalized additive model for location, scale, and shape (GAMLSS) was used (24), as well as the within-population SDS for suprailiac skinfold thickness. Crude sex differences were analyzed by an independent-sample $t$-test (continuous variables) or using Fisher's exact test (categorical variables). Normal distribution was obtained by log-transformation (SAT\%, TAT\%, android fat\%, leptin, and leptin-to-adiponectin ratio (LAR)) or by square root transformation (adiponectin).

All subsequent analyses were stratified by gender. Linear correlation was assessed by Pearson's correlation coefficient. Prediction of VAT\% and SAT\% by each variable was assessed from simple linear regression (adjusted determination coefficient
$\left.\left(R^{2}\right) \times 100\right)$. The agreement between total abdominal fat $\%$ on MRI and android fat\% on DXA evaluation was assessed from a Bland Altman plot with limits of agreement (mean, 95\% confidence interval (CI) ) (25). Difference in SAT\% or VAT\% between early pubertal stage (Tanner stage $<$ B2 in girls and $<\mathrm{G} 2$ in boys) and late pubertal stage (Tanner stage $\geq B 3$ in girls and $\geq G 5$ in boys) was analyzed by means of an independent sample $t$-test. The International Obesity Task Force (IOTF) classification was used to group the participants as underweight (thinness grade $1+2+3$ ), normal weight, or overweight/obese (2). Differences in abdominal fat between the IOTF groups were analyzed using one-way ANOVA with post hoc analyses. All statistical analyses were performed using the Statistical Package for the Social Sciences (SPSS), version 22 (IBM Corp, Armonk, NY, USA). A $P$ value $<0.05$ was considered statistically significant.

\section{Ethics}

The birth cohort study was approved by the local ethics committee (KF 01-030/97/KF 01276357/H-1-2009-074) and the Danish Data Protection Agency (1997-1200-074/2005-41-5545/2010-41-4757). All families gave their informed written consent to participate in the study.

\section{RESULTS}

The mean age (SD) was 12.4 years (0.9) and the mean BMI SDS was 0.02 (1.06). No differences were found in age, BW SDS, BMI SDS, WHtR, WC SDS and suprailiac skinfold SDS between girls and boys (Table 1). Puberty was more advanced in girls when compared with boys, and girls had more adipose tissue than did boys (Tables 1 and 2). According to the MRI results, girls had a higher SAT\% than did boys, but VAT\% was similar (Table 2). As a result, the VAT/SAT ratio was lower in girls compared with that in boys. These sex differences remained significant in analyses stratified by pubertal stage (Supplementary Table S2) (SAT\%: early puberty, relative difference 25\% (95\% CI: 8-45\%), $P<0.01$ 
Table 1. Characteristics of the study population $(n=197)$

\begin{tabular}{|c|c|c|c|c|c|}
\hline & \multicolumn{2}{|c|}{ Boys } & \multicolumn{2}{|r|}{ Girls } & \multirow[b]{2}{*}{$P$-value } \\
\hline & $n$ & & $n$ & & \\
\hline Age (year) & 114 & $12.4(10.5,14.4)$ & 83 & $12.3(10.5,14.1)$ & 0.47 \\
\hline BW SDS & 114 & $0.09(-2.28,2.45)$ & 83 & $0.04(-2.26,2.33)$ & 0.77 \\
\hline Height (cm) & 114 & $158(136.7,179.3)$ & 83 & $156.8(138.6,175.1)$ & 0.42 \\
\hline Height SDS & 114 & $0.10(-2.06,2.25)$ & 83 & $0.32(-2.07,2.72)$ & 0.16 \\
\hline Weight $(\mathrm{kg})^{*}$ & 114 & $45.5(28.9,71.6)$ & 83 & $45.1(29.7,68.4)$ & 0.78 \\
\hline Weight SDS & 114 & $0.02(-2.03,2.07)$ & 83 & $0.29(-1.90,2.48)$ & 0.08 \\
\hline BMI $\left(\mathrm{kg} / \mathrm{m}^{2}\right)^{*}$ & 114 & $18.3(14,24)$ & 83 & $18.4(13.7,24.7)$ & 0.82 \\
\hline BMI SDS & 114 & $-0.07(-2.11,1.97)$ & 83 & $0.15(-2.05,2.35)$ & 0.14 \\
\hline IOTF grade $(\%)^{a}$ & 114 & $10 / 79 / 11$ & 83 & $11 / 81 / 8$ & 0.39 \\
\hline$W C(\mathrm{~cm})^{*}$ & 114 & $66.4(54.9,80.3)$ & 83 & $64.7(53.3,78.4)$ & 0.06 \\
\hline WC SDS & 114 & $0.11(-1.77,1.98)$ & 83 & $0.12(-1.86,2.10)$ & 0.93 \\
\hline WHtR & 114 & $0.42(0.36,0.49)$ & 83 & $0.41(0.34,0.49)$ & 0.11 \\
\hline Suprailiac skinfold $(\mathrm{mm})^{*}$ & 114 & $8.2(3.2,20.8)$ & 83 & $10.3(4.5,23.4)$ & $<0.001$ \\
\hline Suprailiac skinfold (SDS) & 114 & $0.05(-1.73,1.82)$ & 83 & $0.01(-1.72,1.74)$ & 0.79 \\
\hline Pubertal stage $1-5(\%)^{\mathrm{b}}$ & 105 & $22 / 32 / 29 / 15 / 2$ & 81 & $10 / 22 / 27 / 38 / 3$ & 0.003 \\
\hline
\end{tabular}

BMI, body mass index; BW, birth weight; IOTF, International Obesity Task Force; WC, waist circumference; WHtR, waist-to-height ratio.

Results are presented as mean $( \pm 2 \mathrm{SD})$ or geometric mean $( \pm 2 \mathrm{SD})$ for the log-transformed variables marked by $(*)$. Sex-differences were analyzed by independent sample $t$-test (continuous variables) or Fisher's exact test (categorical variables).

aThinness grade 1, 2, and 3/normal weight/overweight, and obese.

bTanner genital stage in boys and tanner breast stage in girls.

and late puberty, relative difference $34 \%$ (13-61\%), $P=0.001)$; VAT\%: early puberty, mean difference $0 \%(0-1 \%), P=0.43$ and late puberty, mean difference $0 \%(-1-1 \%), P=0.93)$. SAT $\%$ increased with pubertal stage in girls and with IOTF classification in both sexes, but no differences in VAT\% were found (Supplementary Table S2).

Overall, VAT\% and SAT\% were positively correlated ( $r=0.32, P=0.003$ in girls and $r=0.49, P<0.001$ in boys). DXA android fat $\%$ and anthropometric measurements were strongly correlated with SAT\% in both boys and girls (Figure 2; Supplementary Fig S3), whereas the correlation with VAT\% was weaker and less consistent (Figure 3; Supplementary Fig S3). In girls, the association between VAT\% and BMI SDS and WC SDS, respectively, did not reach statistical significance. Leptin concentrations and LAR were significantly higher in girls when compared with those in boys, whereas adiponectin concentrations were comparable (Table 2). Leptin was positively correlated with SAT\% and VAT\% in both sexes, but the correlation with VAT\% was weaker than that with SAT $\%$ (Figure 2 and Figure 3, respectively). LAR followed the same pattern as leptin, but the correlation coefficients were lower (SAT\%: $r=0.69$ in girls and $r=0.64$ in boys; VAT: $r=0.19$ in girls and $r=0.35$ in boys). Adiponectin was not correlated with SAT\% or VAT\% (Figure 2 and Figure 3, respectively).

\section{Markers of SAT\% and VAT\% by MRI}

Based on the variance explained in simple regression analyses, the best marker of both SAT\% and VAT\% was DXA android fat\%. (Table 3). Apart from DXA, the best marker of SAT\% and VAT\% was suprailiac skinfold thickness or serum leptin (Table 3). BMI was a better marker of SAT\% than was WHtR or WC, whereas WHtR was a better marker of VAT\% than were BMI and WC. In girls, WHtR was the best marker of VAT\%. Serum adiponectin and age were not important markers of abdominal fat compartments. Puberty explained a minor part of the variance in SAT\% in girls, but this did not reach statistical significance.

Overall, the prediction of SAT\% by any parameter was higher than the prediction of VAT\% (Table 3). Furthermore, the prediction of both SAT\% and VAT\% was generally greater in boys than in girls.

\section{Comparing DXA and MRI}

TAT\% measured by MRI was greater than android fat $\%$ measured by DXA in both sexes (relative difference $\beta=7 \%$ $(3-12 \%)$ in girls and $\beta=18 \%(12-25 \%)$ in boys, both $P<0.01)$. Nevertheless, TAT\% correlated strongly with DXA android fat $\% \quad(r=0.86$ in girls, $r=0.87$ in boys, both $P<0.001)$. Measures obtained using DXA were systematically lower than the measures shown by MRI if abdominal fat $\%$ was low (Supplementary Figure S4). The mean and 95\% limits of agreement were $2 \%$ ( -8 to $11 \%)$, indicating a mean discrepancy between the two methods by up to $10 \%$, depending on the fat percentage.

\section{DISCUSSION}

Within this well-characterized cohort of predominantly normal-weight children and adolescents, the SAT\% varied 
Table 2. Body fat distribution measured by DXA and MRI, and serum adipokines in the study population

\begin{tabular}{|c|c|c|c|c|c|}
\hline & \multicolumn{2}{|r|}{ Boys } & \multicolumn{2}{|r|}{ Girls } & \multirow[b]{2}{*}{$P$-value } \\
\hline & $n$ & & $n$ & & \\
\hline \multicolumn{6}{|l|}{$D X A$} \\
\hline Total fat mass $(\mathrm{g})^{\mathrm{a}}$ & 113 & $7,974(2,666,23,854)$ & 83 & $10,283(4,046,26,139)$ & 0.001 \\
\hline Android fat mass $(\mathrm{g})^{\mathrm{a}}$ & 113 & $469(122,1,801)$ & 83 & $635(197,2,042)$ & 0.001 \\
\hline Total fat $(\%)^{a}$ & 113 & $18(8,40)$ & 83 & $23(12,43)$ & $<0.001$ \\
\hline Total fat (\%) SDS & 113 & $0.04(-0.72,1.81)$ & 83 & $0.18(-1.79,2.16)$ & 0.30 \\
\hline Android fat $(\%)^{a}$ & 113 & $17(6,48)$ & 83 & $24(11,53)$ & $<0.001$ \\
\hline Android fat (\%) SDS & 113 & $0.09(-1.78,1.97)$ & 83 & $0.19(-0.68,2.05)$ & 0.50 \\
\hline \multicolumn{6}{|l|}{ MRl-abdomen } \\
\hline Total abdominal volume $(\mathrm{ml})^{\mathrm{a}}$ & 114 & $4,413(2,707,6,988)$ & 83 & $4,359(2,640,7,198)$ & 0.86 \\
\hline $\operatorname{TAT}(\mathrm{ml})^{\mathrm{a}}$ & 114 & $907(351,2,349)$ & 83 & $1,115(420,2,959)$ & 0.02 \\
\hline $\operatorname{SAT}(\mathrm{ml})^{\mathrm{a}}$ & 114 & $608(202,1,832)$ & 83 & $813(264,2,506)$ & $<0.001$ \\
\hline $\operatorname{VAT}(\mathrm{ml})^{\mathrm{a}}$ & 114 & $289(146,573)$ & 83 & $284(132,611)$ & 0.93 \\
\hline TAT $(\%)^{a}$ & 114 & $21(11,38)$ & 83 & $26(13,49)$ & $<0.001$ \\
\hline SAT $(\%)^{a}$ & 114 & $14(6,30)$ & 83 & $19(8,42)$ & $<0.001$ \\
\hline VAT (\%) & 114 & $7(4,10)$ & 83 & $7(3,10)$ & 0.80 \\
\hline VAT/SAT ratio & 114 & $0.5(0.2,0.8)$ & 83 & $0.4(0.1,0.7)$ & $<0.001$ \\
\hline \multicolumn{6}{|l|}{ Adipokines } \\
\hline Leptin $(\mathrm{ng} / \mathrm{ml})^{\mathrm{a}}$ & 109 & $2.82(0.73,11)$ & 81 & $5.17(0.96,28)$ & $<0.001$ \\
\hline Adiponectin $(\mu \mathrm{g} / \mathrm{ml})^{\mathrm{b}}$ & 109 & $14.22(4.32,29.87)$ & 81 & $12.91(4.69,25.22)$ & 0.13 \\
\hline $\operatorname{LAR}(\mathrm{ng} / \mu \mathrm{g})^{\mathrm{a}}$ & 109 & $0.21(0.04,1.06)$ & 81 & $0.42(0.06,2.94)$ & $<0.001$ \\
\hline
\end{tabular}

from $6 \%$ to $41 \%$ and the VAT\% varied from $3 \%$ to $10 \%$, reflecting the physiological heterogeneity in body composition. Suprailiac skinfold thickness was the single best anthropometric marker of SAT\%, and its anatomical landmark specifically addresses abdominal subcutaneous fat. However, more commonly used simple, cheap, and easily accessible anthropometric measurements such as BMI, WC, and WHtR were also valid markers of SAT\%. These findings are in agreement with those of other studies $(11,26)$ and suggest that suprailiac skinfold thickness could be added to the assessment of body composition in clinical practice and research.

The prediction of VAT\% was difficult, which is in line with previous studies $(10,26-29)$. The poor prediction of VAT\% may be explained by the sparse accumulation and small variance in VAT\% at this age in normal-weight children (10). Obese children tend to have more VAT than do lean children $(10,29,30)$, and the prediction of VAT from anthropometry is less difficult in obese adolescents when compared with lean adolescents (29). In our study, the prediction of VAT\% was greater in boys when compared with girls, perhaps due to the higher VAT/SAT ratio in boys. Most studies demonstrate that
SAT, but not VAT, correlates strongly with anthropometry $(10,27-29,31)$. However, the reported areas and volumes of abdominal fat compartments as well as VAT/SAT ratios in children vary, presumably because of differences in study populations and MRI protocols, which hinder comparison.

Overall, DXA android fat\% was the best marker of both SAT\% and VAT\%. DXA can be performed in a hospital setting and also in population cohorts. In very lean adolescents, DXA underestimated the total abdominal fat $\%$ when compared with MRI, reflecting a bias, which has also been found in other studies $(30,31)$. The 2-dimensional modality of DXA most likely does not allow the measurement of very small amounts of VAT often found in children. In our study, the android region was not the exact same region as measured by MRI, which is expected to introduce some variation but not a systematic bias.

We were able to show an association between abdominal fat and leptin, but not adiponectin. Circulating leptin reflects total fat mass (14) and is a strong marker of SAT\%. Adiponectin is produced by adipocytes and is positively associated with increased insulin sensitivity (32). In obese children, adiponectin is inversely associated with visceral fat, 

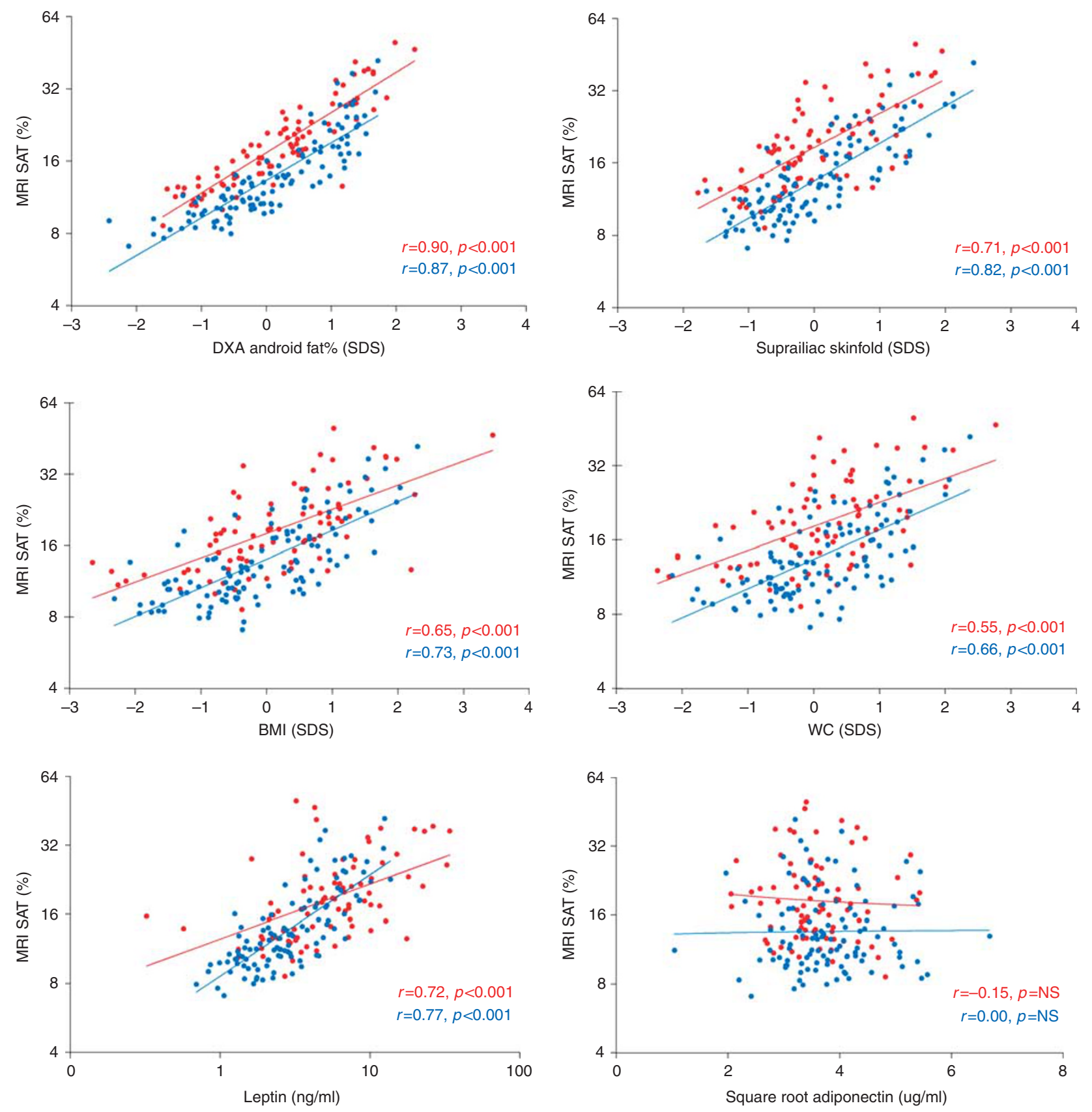

Figure 2. Scatter plots of abdominal subcutaneous adipose tissue percentage (SAT\%, log scale) by MRI versus DXA android fat\% SDS, suprailiac skinfold SDS, BMI SDS, WC SDS, serum leptin (log scale), and adiponectin (square root transformed). Red=girls. Blue=boys. Dots represent individual measurements; lines represent the linear correlation. $r=$ Pearson's correlation coefficient. NS, non-significant.

although evidence is sparse and the association has not been found in lean children $(13,33)$. Because adiponectin and leptin correlate in opposite directions with BMI, their ratio may be superior in reflecting visceral adiposity and the metabolic disturbances related to BMI (34). However, our study did not indicate this. To our knowledge, the association between LAR and VAT\% in children has not previously been reported, and the predictive value of LAR in children is largely unknown and needs further elucidation (35).
It has been demonstrated that the amount of visceral fat increases with age in adolescent boys, but not in girls, and this sex discrepancy may be associated with reproductive hormones $(36,37)$. We did not find an association between VAT\% and pubertal development, which is in line with other studies $(28,29)$. However, longitudinal studies are needed to elucidate changes in abdominal adipose tissue distribution during puberty. Interestingly, in our study, a sexual dimorphism in the VAT/SAT ratio was already apparent in 

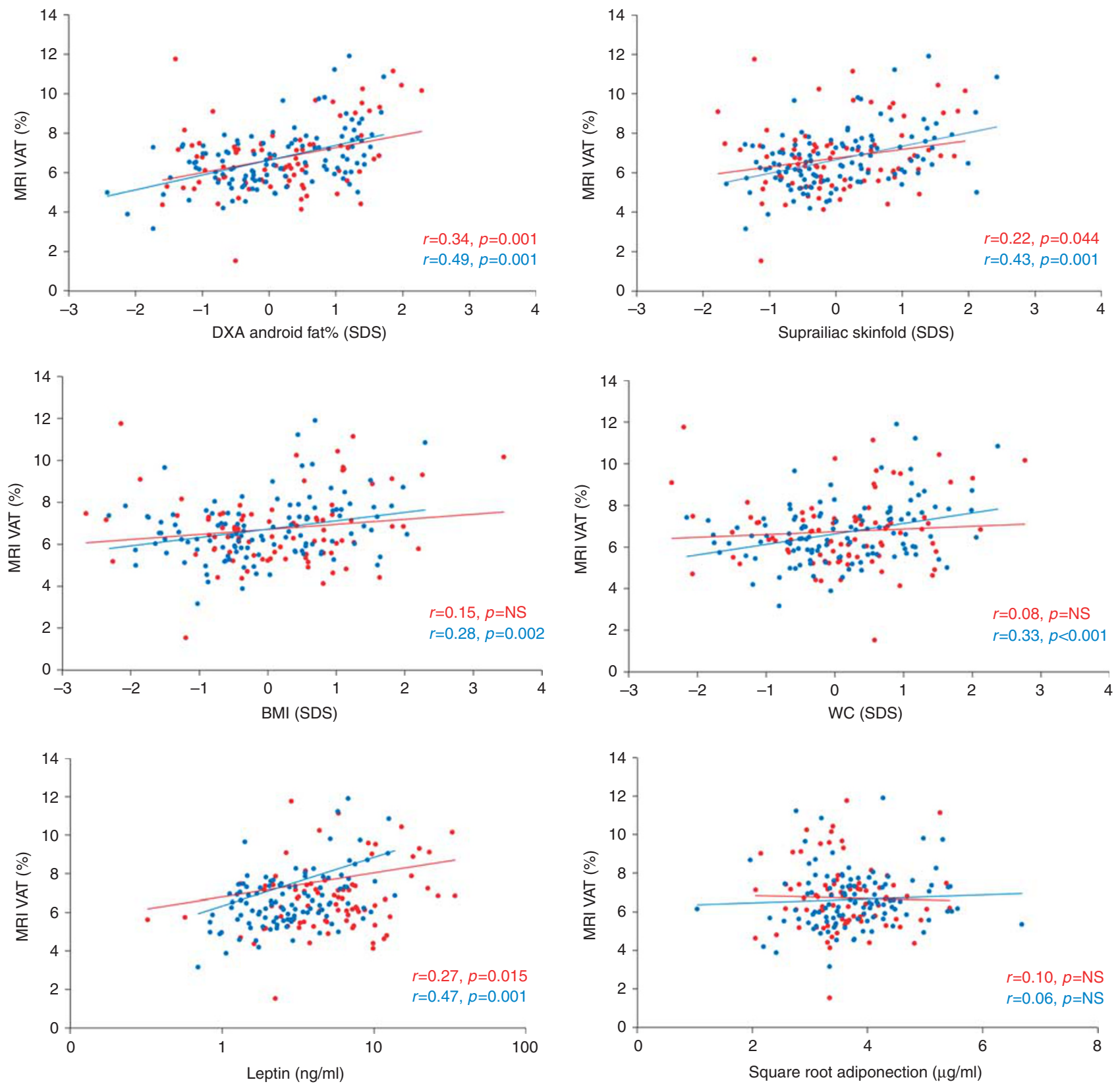

Figure 3. Scatter plots of abdominal visceral adipose tissue percentage (VAT\%) by MRI versus DXA android fat\% SDS, suprailiac skinfold SDS, BMI SDS, WC SDS, serum leptin (log scale), and adiponectin (square root transformed). Red=girls. Blue= boys. Dots represent individual measurements; lines represent the linear correlation. $r=$ Pearson's correlation coefficient. NS, non-significant.

early puberty, with boys having a higher VAT/SAT than did girls. Benfield et al. (10) also reported a higher VAT/SAT ratio in normal-weight boys when compared with girls in adolescence. In the prospective study by Fox et al. (26), the VAT/SAT ratio increased from the age of 11 to 13 in boys, but decreased in girls. The amount of VAT appears to be low in normal-weight children. Although the clinical implication of measuring VAT in normal-weight children is unclear, an increased VAT-to-SAT ratio may lead to an increased disease risk because subcutaneous fat, in contrast to visceral fat, has metabolically protective effects (36). Further investigation is required to understand whether the sexual dimorphism in the VAT/SAT ratio during early adolescence is a predisposition to the higher risk of cardio-vascular disease in adult men compared with women.

Our study has some limitations. The selection of children for MRI was based on a high frequency of prior visits, which may introduce selection bias. However, birth weight SDS, distribution of SES, and BMI SDS at pubertal follow-up were similar to those of the remaining cohort (data not shown). Because all children were Caucasian and predominantly of normal weight, our results can be generalized only to similar 
Table 3. Variance of SAT\% and VAT\% measured by MRI explained by anthropometry, serum adipokines, puberty, or age as individual variables

\begin{tabular}{|c|c|c|c|c|c|c|c|c|}
\hline & \multicolumn{4}{|c|}{ SAT (\%) } & \multicolumn{4}{|c|}{ VAT\% } \\
\hline & \multicolumn{2}{|c|}{ Boys } & \multicolumn{2}{|c|}{ Girls } & \multicolumn{2}{|c|}{ Boys } & \multicolumn{2}{|c|}{ Girls } \\
\hline & Variance $(\%)^{a}$ & $P$-value & Variance $(\%)^{a}$ & $P$ value & Variance $(\%)^{a}$ & $P$ value & Variance $(\%)^{a}$ & $P$ value \\
\hline DXA android fat $\%$ SDS & 76 & $<0.001$ & 81 & $<0.001$ & 23 & $<0.001$ & 11 & 0.001 \\
\hline Suprailiac skinfold SDS & 65 & $<0.001$ & 49 & $<0.001$ & 16 & $<0.001$ & 7 & 0.009 \\
\hline Leptin & 59 & $<0.001$ & 51 & $<0.001$ & 21 & $<0.001$ & 6 & 0.02 \\
\hline BMI SDS & 53 & $<0.001$ & 41 & $<0.001$ & 7 & 0.002 & 1 & 0.17 \\
\hline WHtR & 48 & $<0.001$ & 31 & $<0.001$ & 15 & $<0.001$ & 8 & 0.007 \\
\hline WC SDS & 43 & $<0.001$ & 30 & $<0.001$ & 10 & $<0.001$ & 1 & 0.79 \\
\hline LAR & 40 & $<0.001$ & 47 & $<0.001$ & 12 & $<0.001$ & 2 & 0.10 \\
\hline Height SDS & 4 & 0.03 & 0 & 0.43 & 1 & 0.58 & 3 & 0.05 \\
\hline Puberty $^{\text {b }}$ & 0 & 0.25 & 3 & 0.08 & 0 & 0.42 & 1 & 0.18 \\
\hline Adiponectin & 1 & 0.99 & 1 & 0.17 & 1 & 0.55 & 0 & 0.39 \\
\hline Age & 0 & 0.35 & 0 & 0.25 & 1 & 0.86 & 1 & 0.74 \\
\hline
\end{tabular}

BMI, body mass index; LAR, leptin-to-adiponectin ratio; WC, waist circumference; WHtR, waist-to-height ratio.

${ }^{a}$ Adjusted-determination coefficient $\left(R^{2}\right) \times 100$.

${ }^{b}$ Early vs. late puberty (Tanner B or G $<3$ vs. Tanner B or G QUOTE 3, in girls and boys, respectively).

populations and not to obese children. We do not have fasting blood samples. However, serum adiponectin is unaffected by prolonged fasting (38) and although serum leptin declines during prolonged fasting, serum leptin reflects fat mass rather than energy homeostasis (39). Furthermore, serum levels of adipokines were comparable to fasting levels reported in normal-weight adolescents (13,14,35). A major strength of this study is the large population size in relation to the extensive MRI protocol.

\section{SUMMARY AND CONCLUSION}

Using multi-slice MRI of the abdomen, this study demonstrated that within predominantly normal-weight young adolescents VAT\% does not differ between girls and boys, whereas SAT\% is higher among girls than among boys, independently of puberty. Whether the consequent gender difference in the VAT-to-SAT ratio is associated with an increased risk for cardio-metabolic disease among adult men compared with women needs further investigation.

In normal-weight children, DXA android fat $\%$ tends to underestimate total abdominal fat $\%$ by MRI. However, DXA android fat $\%$ is a stronger marker of SAT\% and VAT\% than are simple anthropometry and circulating leptin levels.

Assessment of VAT in normal-weight children is challenging. Neither DXA android fat\% nor anthropometry and circulating adiponectin levels are valid markers of VAT\%. This may relate to the sparse accumulation of VAT at this age. Thus, simple markers of VAT in normal-weight children are still lacking.

SAT\% can be measured by either DXA or simple anthropometry and is associated with circulating leptin levels. Suprailiac skinfold thickness was the strongest anthropometric marker of SAT\%, and this measurement may be implemented more frequently in research and clinical practise.

\section{SUPPLEMENTARY MATERIAL}

Supplementary material is linked to the online version of the paper at http://www.nature.com/pr

\section{AUTHOR CONTRIBUTIONS}

J.T.: study design, data collection, data interpretation, literature search, initial analyses, and drafting of the manuscript, tables, and figures. C.P.H., A.M., M.G.M., and C.W.-V.: study design, data collection, and data interpretation. R.B.J.: study design, supervision of data analyses, and interpretation of data. A.J. and K.M.M.: study conceptualization and design, supervision of data analyses, and interpretation of data. A.N.C., R.L., E.F., J. W.H., and T.N.B.: study design and data analyses. All authors revised the manuscript critically, approved the final version as submitted, and agreed to be accountable for all aspects of the work.

\section{STATEMENT OF FINANCIAL SUPPORT}

The Danish Agency for Science, Technology and Innovation (09-067180), The Danish Environmental Protection Agency (MST-621-00065) and Ministry of Higher Education and Science ((DFF-1331-00133).

Disclosure: The authors declare no conflict of interest.

\section{REFERENCES}

1. Reilly JJ, Kelly J. Long-term impact of overweight and obesity in childhood and adolescence on morbidity and premature mortality in adulthood: systematic review. Int J Obes 2011;35:891-8.

2. Cole TJ, Lobstein T. Extended international (IOTF) body mass index cutoffs for thinness, overweight and obesity. Pediatr Obes 2012;7:284-94.

3. Gishti O, Gaillard R, Durmus B, et al. BMI, total and abdominal fat distribution, and cardiovascular risk factors in school-age children. Pediatr Res 2015;77:710-8.

4. Abraham TM, Pedley A, Massaro JM, Hoffmann U, Fox CS. Association between visceral and subcutaneous adipose depots and incident cardiovascular disease risk factors. Circulation 2015;132:1639-47.

5. Thomas EL, Frost G, Taylor-Robinson SD, Bell JD. Excess body fat in obese and normal-weight subjects. Nutr Res Rev 2012;25:150-61. 


\section{Articles | Tinggaard et al.}

6. Sung YJ, Perusse L, Sarzynski MA, et al. Genome-wide association studies suggest sex-specific loci associated with abdominal and visceral fat. Int J Obes 2016;40:662-74.

7. $\mathrm{Hu} \mathrm{HH}$, Nayak KS, Goran MI. Assessment of abdominal adipose tissue and organ fat content by magnetic resonance imaging. Obes Rev 2011;12: e504-15.

8. Sharma AK, Metzger DL, Daymont C, Hadjiyannakis S, Rodd CJ. LMS tables for waist-circumference and waist-height ratio Z-scores in children aged 5-19 y in NHANES III: association with cardio-metabolic risks. Pediatr Res 2015;78:723-9.

9. Zimmet P, Alberti G, Kaufman F, et al. The metabolic syndrome in children and adolescents. Lancet 2007;369:2059-61.

10. Benfield LL, Fox KR, Peters DM, et al. Magnetic resonance imaging of abdominal adiposity in a large cohort of British children. Int J Obes 2008;32:91-9.

11. Goran MI, Gower BA, Treuth M, Nagy TR. Prediction of intra-abdominal and subcutaneous abdominal adipose tissue in healthy pre-pubertal children. Int J Obes Relat Metab Disord 1998;22:549-8.

12. Mokha JS, Srinivasan SR, Dasmahapatra P, et al. Utility of waist-to-height ratio in assessing the status of central obesity and related cardiometabolic risk profile among normal weight and overweight/obese children: the Bogalusa Heart Study. BMC Pediatr 2010;10:73.

13. Bacha F, Saad R, Gungor N, Arslanian SA. Adiponectin in youth: relationship to visceral adiposity, insulin sensitivity, and beta-cell function. Diabetes Care 2004;27:547-2.

14. Schoppen S, Riestra P, Garcia-Anguita A, et al. Leptin and adiponectin levels in pubertal children: relationship with anthropometric variables and body composition. Clin Chem Lab Med 2010;48:707-11.

15. Wohlfahrt-Veje C, Tinggaard J, Winther K, et al. Body fat throughout childhood in 2647 healthy Danish children: agreement of BMI, waist circumference, skinfolds with dual X-ray absorptiometry. Eur J Clin Nutr 2014;68:664-70.

16. Marsal K, Persson PH, Larsen T, Lilja H, Selbing A, Sultan B. Intrauterine growth curves based on ultrasonically estimated foetal weights. Acta Paediatr 1996;85:843-8.

17. Mouritsen A, Johansen ML, Wohlfahrt-Veje C, et al. Determination of adrenal volume by MRI in healthy children: associations with age, body size, pubertal stage and serum levels of adrenal androgens. Clin Endocrinol 2014;81:183-9.

18. Fugl L, Hagen CP, Mieritz MG, et al. Glandular breast tissue volume by magnetic resonance imaging in 100 healthy peripubertal girls: evaluation of clinical Tanner staging. Pediatr Res 2016;80:526-30.

19. Hagen CP, Mouritsen A, Mieritz MG, et al. Circulating AMH reflects ovarian morphology by magnetic resonance imaging and $3 \mathrm{D}$ ultrasound in 121 healthy girls. J Clin Endocrinol Metab 2015;100:880-90.

20. Mosbech TH, Pilgaard K, Vaag A, Larsen R Automatic Segmentation of Abdominal Adipose Tissue in MRI. Berlin, Heidelberg: Springer-Verlag, 2011:501-511.

21. Li K, Wu X, Chen DZ, Sonka M. Optimal surface segmentation in volumetric images - a graph-theoretic approach. IEEE Trans Pattern Anal Mach Intell 2006;28:119-34.
22. Otsu N. A threshold selection methods from gray-level histograms. IEEE Trans Syst Man Cybernet 1979; 9:62-6.

23. Tinggaard J, Aksglaede L, Sorensen K, et al. The 2014 Danish references from birth to 20 years for height, weight and body mass index. Acta Paediatr 2014;103:214-4.

24. Rigby RA, Stasinopoulos DM. Generalized additive models for location, scale and shape. Appl Stat 2005;54:507-4.

25. Bland JM, Altman DG. Measuring agreement in method comparison studies. Stat Methods Med Res 1999;8:135-60.

26. Fox KR, Peters DM, Sharpe P, Bell M. Assessment of abdominal fat development in young adolescents using magnetic resonance imaging. Int J Obes Relat Metab Disord 2000;24:1653-9.

27. Barreira TV, Broyles ST, Gupta AK, Katzmarzyk PT. Relationship of anthropometric indices to abdominal and total body fat in youth: sex and race differences. Obesity 2014;22:1345-50.

28. Goodwin K, Syme C, Abrahamowicz M, et al. Routine clinical measures of adiposity as predictors of visceral fat in adolescence: a population-based magnetic resonance imaging study. PLoS ONE 2013;8: e79896.

29. Koren D, Marcus CL, Kim C, et al. Anthropometric predictors of visceral adiposity in normal-weight and obese adolescents. Pediatr Diabet 2013;14:575-84.

30. Siegel MJ, Hildebolt CF, Bae KT, Hong C, White NH. Total and intraabdominal fat distribution in preadolescents and adolescents: measurement with MR imaging. Radiology 2007;242:846-56.

31. Karlsson AK, Kullberg J, Stokland E, et al. Measurements of total and regional body composition in preschool children: a comparison of MRI, DXA, and anthropometric data. Obesity 2013;21:1018-24.

32. Pyrzak B, Ruminska M, Popko K, Demkow U. Adiponectin as a biomarker of the metabolic syndrome in children and adolescents. Eur J Med Res 2010;15:147-51.

33. Asayama K, Hayashibe H, Dobashi K, et al. Decrease in serum adiponectin level due to obesity and visceral fat accumulation in children. Obes Res 2003;11:1072-9.

34. Finucane FM, Luan J, Wareham NJ, et al. Correlation of the leptin: adiponectin ratio with measures of insulin resistance in non-diabetic individuals. Diabetologia 2009;52:2345-9.

35. Diamond FB Jr, Cuthbertson D, Hanna S, Eichler D. Correlates of adiponectin and the leptin/adiponectin ratio in obese and non-obese children. J Pediatr Endocrinol Metab 2004;17:1069-75.

36. Palmer BF, Clegg DJ. The sexual dimorphism of obesity. Mol Cell Endocrinol 2015;402:113-9.

37. Satake E, Nakagawa Y, Kubota A, Saegusa H, Sano S, Ohzeki T. Age and sex differences in fat distribution in non-obese Japanese children. J Pediatr Endocrinol Metab 2010;23:873-8.

38. Merl V, Peters A, Oltmanns KM, et al. Serum adiponectin concentrations during a 72-hour fast in over- and normal-weight humans. Int J Obes 2005;29:998-1001.

39. Weigle DS, Duell PB, Connor WE, Steiner RA, Soules MR, Kuijper JL. Effect of fasting, refeeding, and dietary fat restriction on plasma leptin levels. J Clin Endocrinol Metab 1997;82:561-5. 